https://doi.org/10.15407/ujpe63.6.479

V.S. OVECHKO, V.P. MYHASHKO

Taras Shevchenko National University of Kyiv

(4g, Prosp. Academician Glushkov, Kyiv 03022, Ukraine; e-mail: ovs@univ.kiev.ua)

\title{
SPECTRAL PARTICULARITIES \\ OF FEMTOSECOND OPTICAL PULSES PROPAGATING IN DISPERSIVE MEDIUM ${ }^{1}$
}

\section{Introduction}

The traditional theory and typical experiments are based on the consideration of "narrowband signals" $[1-5]$. At the same time, the femtosecond time durations of optical pulses do not fit such approximation. Here, the objective is twofold: (1) to study the ultra wideband optical signal propagation in a dispersive medium; (2) to revise some well-known notions for the "slowly varying envelope approximation". We consider the Lorentz-model of a medium with regard for the distinction between an active electric pole $\overline{\mathbf{E}}_{a}$ and an average macroscopic pole $\overline{\mathbf{E}}$ in a medium $[1,3]$. We use the orthonormalized series of EWP-functions (elementary wave packets) for describing the electromagnetic pulses without restriction on their durations [6]. We have solved the wave equation describing the optical pulse propagation without any approximations. The solution of the wave equation is used for the investigation of lower-order EWP-modes. Particular interests of the investigation of the solution are due to the inconclu-

(C) V.S. OVECHKO, V.P. MYHASHKO, 2018

ISSN 2071-0194. Ukr. J. Phys. 2018. Vol. 63, No. 6 sive insight on the Beer-Lambert law of absorption, Rayleigh approximation for phase and group propagation velocities, Sommerfeld and Brillouin points of view on the information velocity (signal velocity), time-domain spectroscopy of superwideband optical pulses, etc.

\section{Solving the Wave}

\section{Equation for a Dispersive Medium}

We seek a solution for the scalar wave equation in a nonmagnetic $(\mu=1)$ dispersive medium with boundary conditions

$\frac{\partial^{2} E(z, t)}{\partial z^{2}}-\frac{1}{c^{2}} \frac{\partial^{2} E(z, t)}{\partial t^{2}}=\frac{4 \pi}{c^{2}} \frac{\partial^{2} P(z, t)}{\partial t^{2}}$,

where $E(z, t)$ is the electric component,

$P(z, t)=\int_{0}^{\infty} \chi\left(t^{\prime}\right) E\left(t-t^{\prime}\right) d t^{\prime}$

${ }^{1}$ The paper was presented at the XXIII Galyna Puchkovska International School-Seminar "Spectroscopy of Molecules and Crystals". 
is the polarization, $E(0, t)=E_{0}(t)$ is an input optical pulse, and $\frac{\partial E}{\partial z}(0, t)=E_{1}(t)$ is the boundary condition. Equation (1) in partial derivatives can be simplified by the Fourier-integral decomposition [7]

$E(z, \omega)=\frac{1}{\sqrt{2 \pi}} \int_{-\infty}^{+\infty} E(z, t) e^{-i \omega t} d t$.

As a result, we get the equation with total derivatives

$\frac{\partial^{2} E(z, \omega)}{\partial z^{2}}-\frac{\omega^{2}}{c^{2}} \varepsilon(\omega) E(z, \omega)=0$,

$E(0, \omega)=E_{0}(\omega)$,

$\frac{\partial E(0, \omega)}{\partial z}=E_{1}(\omega)$

where $\varepsilon(\omega)=1+4 \pi \chi(\omega)$ is the dielectric susceptibility. Now, let us calculate $E_{1}(\omega)$. For this purpose, we write down Maxwell's equations

$\frac{\partial B}{\partial t}=\frac{\partial H}{\partial t}=-c \frac{\partial E}{\partial z}$,

$H=\sqrt{\varepsilon} E$.

From (9) and (10), we get

$\frac{\partial E}{\partial z}=-\frac{\sqrt{\varepsilon}}{c} \frac{\partial E}{\partial t}$.

Let us separate the $\operatorname{Re}\left(E_{r}(z, \omega)\right)$ and $\operatorname{Im}\left(E_{i}(z, \omega)\right)$ parts of Eq. (6). We get a system of two inhomogeneous equations with fixed coefficients:

$\frac{d^{2} E_{r}(z, \omega)}{d z^{2}}+\frac{\omega^{2}}{c^{2}} \times$

$\times\left(n^{2}-\kappa^{2}\right) E_{r}(z, \omega)+2 n \kappa \frac{\omega^{2}}{c^{2}} E_{i}(z, \omega)=0$,

$\frac{d^{2} E_{i}(z, \omega)}{d z^{2}}+\frac{\omega^{2}}{c^{2}} \times$

$\times\left(n^{2}-\kappa^{2}\right) E_{i}(z, \omega)-2 n \kappa \frac{\omega^{2}}{c^{2}} E_{r}(z, \omega)=0$

$\varepsilon=n^{2}-\kappa^{2}-2 i n \kappa$,

$n$ is the refractive index, and $\kappa$ is the absorption coefficient. Because $\partial E_{0} /\left.\partial t\right|_{z=0}$ is Re, the boundary conditions for $E(z, \omega)$ are determined by means of the cosine Fourier transformation. Let us solve system (12), (13) by the Euler method in order to present

$E_{r}(z, \omega)=\alpha e^{\lambda z}, E_{i}(z, \omega)=\beta e^{\lambda z}$ and to find the characteristic numbers $\lambda_{j}$. Applying the boundary conditions for $E_{r}(z, \omega)$, we get

$$
\begin{aligned}
& E(z, t)=\sqrt{\frac{2}{\pi}} \int_{0}^{\infty} \exp [-\kappa \omega z / c] \cos [\omega t] \times \\
& \times\left(E_{0}(\omega) \cos \left[n \frac{\omega}{c} z\right]-\frac{E_{1}(\omega)}{\omega} \sin \left[n \frac{\omega}{c} z\right]\right) d \omega,
\end{aligned}
$$

where

$E_{0}(\omega)=\left.\sqrt{\frac{2}{\pi}} \int_{0}^{T} E\right|_{z=0} \cos [\omega t] d t$,

$E_{1}(\omega)=\left.\sqrt{\frac{2}{\pi}} \int_{0}^{T} \frac{\partial E}{\partial t}\right|_{z=0} \cos [\omega t] d t=$

$=\omega \sqrt{\frac{2}{\pi}} \int_{0}^{T} E_{\left.\right|_{z=0}} \sin [\omega t] d t$,

and $T$ is the duration of an input optical pulse.

We do not consider the Fresnel reflectance in formula (15). First of all, we check Eqs. (6), (7) for the well-known solution, for example, for the outer space. Since $n=1, \kappa=0$, we have

$E(z, t)=\sqrt{\frac{2}{\pi}} \int_{0}^{\infty}\left(E_{0}(\omega) \cos \left[\frac{\omega}{c} z\right]-\right.$

$\left.-\frac{E_{1}(\omega)}{\omega} \sin \left[\frac{\omega}{c} z\right]\right) \cos [\omega t] d \omega$.

Let us consider zero-order even EWP-pulses [6]. We have

$E(t)=A\left(\cos \left[\pi \frac{t}{T}\right]-\cos \left[3 \pi \frac{t}{T}\right]\right), \quad 0 \leq t \leq T$.

To simplify the calculation, we use the standardized variables: $t \equiv t / T, \omega \equiv \omega T, z / c T, A=1$. Then Eq. (11) transforms to

$E(z, t)=\sqrt{\frac{2}{\pi}} \int_{0}^{\infty}\left(E_{0 C}(\omega) \cos [\omega z]-\right.$

$\left.-E_{0 S}(\omega) \sin [\omega z]\right) \cos [\omega t] d \omega$

where

$E_{0}(\omega)=\frac{8 \sqrt{2} \pi^{3 / 2} \omega \sin [\omega]}{9 \pi^{4}-10 \pi^{2} \omega^{2}+\omega^{4}}$

ISSN 2071-0194. Ukr. J. Phys. 2018. Vol. 63, No. 6 
$E_{1}(\omega)=-\frac{8 \sqrt{2} \pi^{3 / 2} \omega(1+\cos [\omega])}{9 \pi^{4}-10 \pi^{2} \omega^{2}+\omega^{4}}$.

The results of calculations of $E(z, t)$ and $E(z, \omega)$ as functions of the distance $z$ are shown in Fig. 1. The results are close to the expected one.

One can see that the spectral amplitude with $\omega=0$ is equal to zero. We can calculate analytically that

$I=\sqrt{\frac{2}{\pi}} \int_{0}^{\infty}\left(E_{0 C}(\omega) \cos [\omega z]-\right.$

$\left.-E_{0 S}(\omega) \sin [\omega z]\right) d \omega \equiv 0$,

which satisfies the restriction on the spectral amplitude $E(\omega=0, z)$ of radio signals (EWP-pulses). Then we will make such testing for the Lorentz model of a medium.

\section{Lorentz Model for a Dispersive Medium}

Before passing to the calculation of the optical pulse spreading through a dispersive medium, let us briefly examine the Lorentz model. Despite a great age of the theory, this model stays in order thanks to its simplicity and recognition among experimenters $[1$, 3]. Along with the basic consideration of the medium as an ensemble of noninteracting oscillators, we take the difference between the average $E(t)$ and acting $E_{a}(t)$ amplitudes into account:

$E_{a}(t)=E(t)+\frac{4 \pi}{c} P$.

This relation allows us to investigate not only rarefied media. As is shown (see, e.g., [3]),

$$
\begin{aligned}
& \varepsilon(\omega)=1+\frac{\omega_{p}^{2}\left(\widetilde{\omega}_{0}^{2}-\omega^{2}\right)}{\left(\widetilde{\omega}_{0}^{2}-\omega^{2}\right)^{2}+\omega^{2} y^{2}}+ \\
& +i \frac{\omega_{p}^{2} y \omega}{\left(\widetilde{\omega}_{0}^{2}-\omega^{2}\right)^{2}+\omega^{2} y^{2}},
\end{aligned}
$$

where $\omega_{p}^{2}=4 \pi N e^{2} / m, \widetilde{\omega}_{0}^{2}=\omega_{0}^{2}-\omega_{p}^{2} / 3, y$ is a damping coefficient, $N$ is the concentration of oscillators. The last step is the introduction of $n(\omega), \kappa(\omega)$ $n(\omega)+i \kappa(\omega)=\sqrt{\varepsilon(\omega)}=\sqrt{\varepsilon^{\prime}+i \varepsilon^{\prime \prime}}$. Then

$n(\omega)=\left(\left(\left(\varepsilon^{\prime 2}+\varepsilon^{\prime \prime 2}\right)^{1 / 2}+\varepsilon^{\prime}\right) / 2\right)^{1 / 2}$,

$\kappa(\omega)=\left(\left(\left(\varepsilon^{\prime 2}+\varepsilon^{\prime \prime 2}\right)^{1 / 2}-\varepsilon^{\prime}\right) / 2\right)^{1 / 2}$,
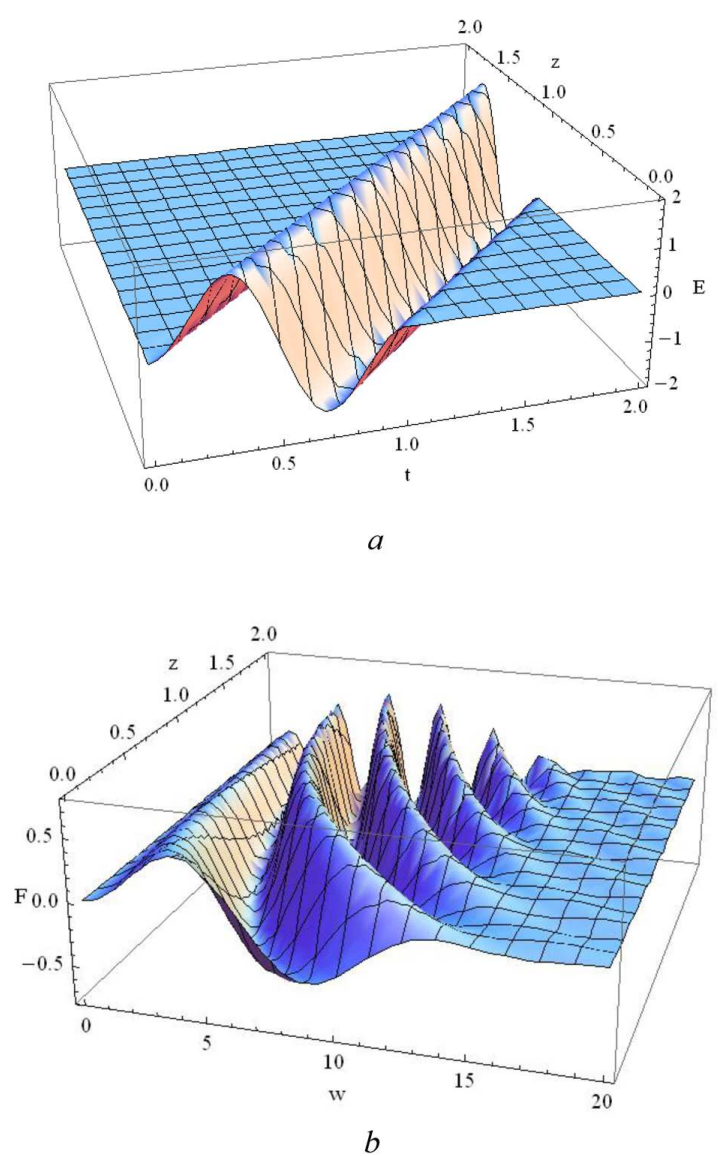

Fig. 1. EWP-pulse $E_{2}(z, t)$ of the zero order $(a)$ and its spectrum $E_{2}(z, \omega)(b): z \equiv z / C T, t \equiv t / T$

where

$\varepsilon^{\prime}=1+\frac{\omega_{p}^{2}\left(\widetilde{\omega}_{0}^{2}-\omega^{2}\right)}{\left(\widetilde{\omega}_{0}^{2}-\omega^{2}\right)^{2}+\omega^{2} y^{2}}$,

$\varepsilon^{\prime \prime}=\frac{\omega_{p}^{2} \omega y}{\left(\widetilde{\omega}_{0}^{2}-\omega^{2}\right)^{2}+\omega^{2} y^{2}}$.

Formulae (21) and (22) can be normalized like (18): $\omega_{p} \equiv \omega_{p} T, y \equiv y T, \omega_{0} \equiv \omega_{0} T$. So, Eq. (15) together with (26)-(27) and the parameters of an input pulse $\left(E_{0}(\omega)\right.$ and $\left.E_{1}(\omega)\right)$ form the solution for an optical pulse, which propagates through a dispersive medium. In what follows, we will study particularities of the propagation of EWP-pulses. Typical normalized frequency dependences for $n(\omega), \kappa(\omega)$, and $E_{0}(\omega)$ are shown in Fig. 2.

One can see that the one-resonance medium cannot affect an ultrawidebaund EWP-pulse of the zero order as a whole. Because of this, some peculiarities 


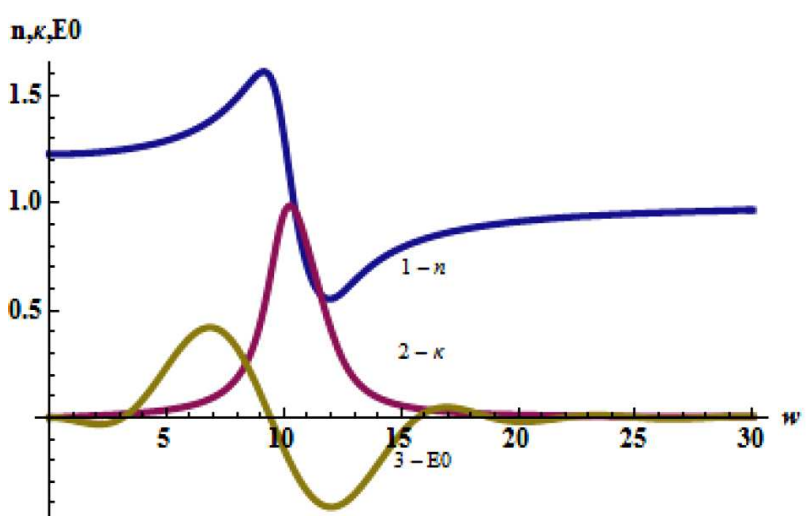

Fig. 2. Refractive index $n(\omega)(1)$, absorption coefficient $\kappa(\omega)$ (2), signal spectrum $E_{2}(z, \omega)(3): \omega_{p}=2, \omega_{0}=10, y=2$
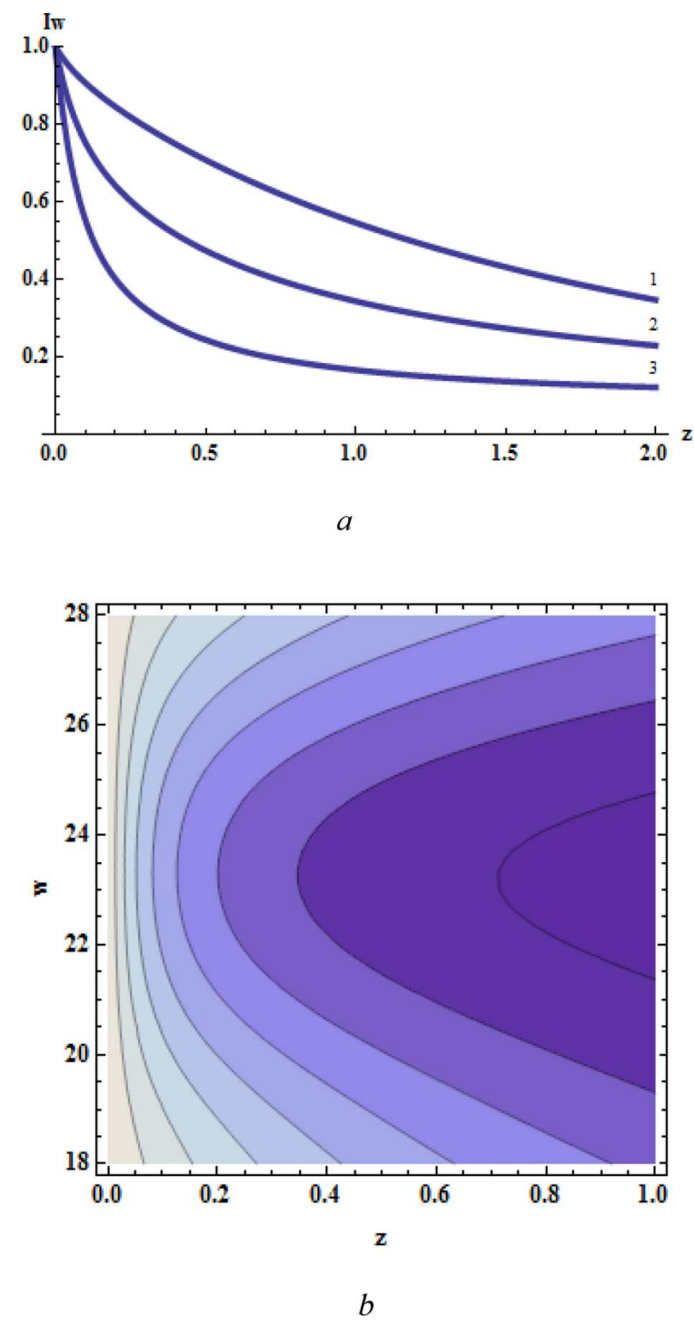

Fig. 3. Signal energy $I_{6 \omega}(z)(a)$ for $\omega_{0}: 15$ (1); 27 (2); 23.2 (3) and contour dependences $I_{\omega}\left(z, \omega_{0}\right)=C_{1}$ for $\omega_{p}=2, y=2$ arise in the propagation of EWP-pulses, as it will be shown in the next section. The amplitude of any spectral component satisfies the Beer-Lambert law. At the same time, the energy of an optical pulse $I_{\omega}(z)$ has a more complicated dependence on $z$, as one can see in Fig. 3, a:

$I_{\omega}(z)=\int_{0}^{\infty} E^{2}(\omega, z) d \omega$.

The two-coordinate dependence of the pulse energy on $z$ and the resonant frequency $\omega_{0}$ is shown in Fig. $3, b$. The amplitude scale in the contour graphs (Figs. 3, 6, 8, and 10) is determined by the operator MashRange in such a way that the amplitudes of two neighboring strips differ by $(100 / N) \%$, where $N$ is the total number of strips. We have

$$
\begin{aligned}
& I_{\omega}\left(z, \omega_{0}\right)=\int_{0}^{\infty} e^{-\kappa\left(\omega, \omega_{0}\right) \omega z}\left(E_{0 C}(\omega) \times\right. \\
& \left.\times \cos \left[n\left(\omega, \omega_{0}\right) \omega z\right]-E_{0 S}(\omega) \sin \left[n\left(\omega, \omega_{0}\right) \omega z\right]\right) d \omega .
\end{aligned}
$$

As one would expect, the spectral components differ from one another by the extinction coefficient (Fig. 3). Superwideband optical signals do not obey the Beer-Lambert law.

\section{EWP-Pulse Propagation Through a One-Resonance Dispersive Medium}

In this section, we study an EWP-pulse of the third order

$$
\begin{aligned}
& E_{6}(t)=(6)^{-1 / 2}(\cos [2 \pi t]+\cos [4 \pi t]+ \\
& +\cos [6 \pi t]-3 \cos [8 \pi t]), \quad 0 \leq t \leq 1, \quad A=1 .
\end{aligned}
$$

The cosine Fourier transform of the pulse shape (32) reads

$$
\begin{aligned}
& E_{0}(\omega)=-\frac{8 \pi^{3 / 2} \omega}{\sqrt{3}} \times \\
& \times \frac{\left(5408 \pi^{4}-700 \pi^{2} \omega^{2}+17 \omega^{4}\right)(\sin [\omega])}{\left(4 \pi^{2}-\omega^{2}\right)\left(16 \pi^{2}-\omega^{2}\right)\left(36 \pi^{2}-\omega^{2}\right)\left(64 \pi^{2}-\omega^{2}\right)},
\end{aligned}
$$

$0 \leq \omega \leq \infty$

$$
\begin{aligned}
& E_{1}(\omega)=\frac{8 \pi^{3 / 2} \omega^{2}}{\sqrt{3}} \times \\
& \times \frac{\left(5408 \pi^{4}-700 \pi^{2} \omega^{2}+17 \omega^{4}\right)(-1+\cos [\omega])}{\left(4 \pi^{2}-\omega^{2}\right)\left(16 \pi^{2}-\omega^{2}\right)\left(36 \pi^{2}-\omega^{2}\right)\left(64 \pi^{2}-\omega^{2}\right)},
\end{aligned}
$$

$0 \leq \omega \leq \infty$.

ISSN 2071-0194. Ukr. J. Phys. 2018. Vol. 63, No. 6 


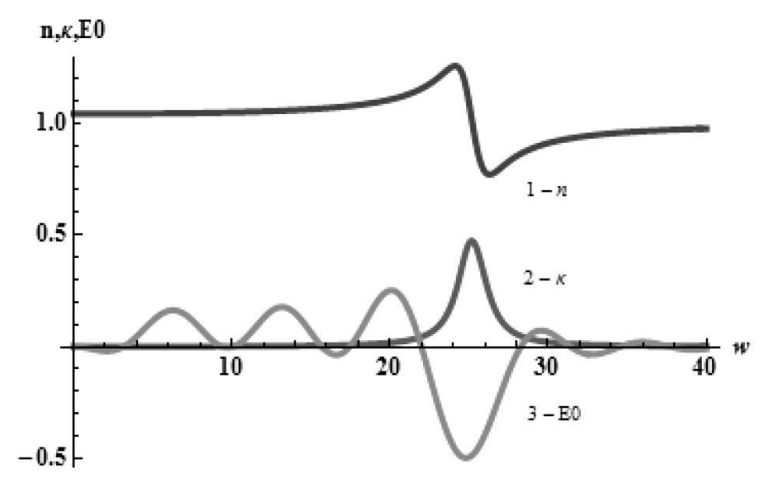

Fig. 4. Refractive index $n(\omega)(1)$, absorption coefficient $\kappa(\omega)$ (2), signal spectrum $E_{6}(\omega)(3): \omega_{p}=2, \omega_{0}=25, y=2$

Let us investigate the time and frequency dependences for aa pulse on the distance $z$, when the resonant frequency of a matter $\omega_{0}$ is near the maximum of the function $E_{0}(\omega)(25): \omega_{0}=25$. The dependences for $n(\omega), \kappa(\omega)$, and $E_{0}(\omega)$ are shown below (Fig. 4). A part of the spectrum $\left(\omega<\omega_{0}\right)$ has $n>1$, another part $\left(\omega>\omega_{0}\right)$ has $n<1$, the part $(24 \leq \omega \leq 26)$ being a region of the anomalous dispersion $(d n / d \omega<0$. The pulse envelope, its position, and the spectrum depend on the distance $z$ and the medium parameters: $\omega_{p}, \omega_{0}, y$.

To understand the pulse propagation in details, let us consider: (1) behavior of the low- and highfrequency spectra; (2) parameters of the so-called "forerunner"; (3) velocity of the EWP-pulse. In Fig. 5, the influence of high, medium, and low frequencies are shown. First of all, the EWP-pulse is formed by the interference of spectral components of all three parts. Low-frequency components brings the basic energy in pulse (Fig. 5, a). High frequencies execute the action as a shot pulse for a resonant contour (Fig. 5, b). After the leading and back edges of the pulse, there are relaxation oscillations. The duration of the front of pulses $t_{p} \simeq 0.1,2 \pi / \omega_{0} \simeq 0.25$.

The anomalous dispersion region must have "anomalous velocities". One can see (Fig. 5, c) that a local change of the amplitude with the distance $z$ takes place almost instantly. But we cannot define the velocity for a "part of the pulse". For this purpose, let us calculate the contour dependences $E(z, t)=C_{i}$, $C_{i}$ are constants. We do it for two medium frequency $\omega_{0}$ disposals: 1) $\left.\omega_{0}=20<25,2\right) \omega_{0}=30>25$. The corresponding dependences are shown in Fig. 6 .

At the same time, we draw up the lines, which correspond to the light velocity in vacuum $(C=$
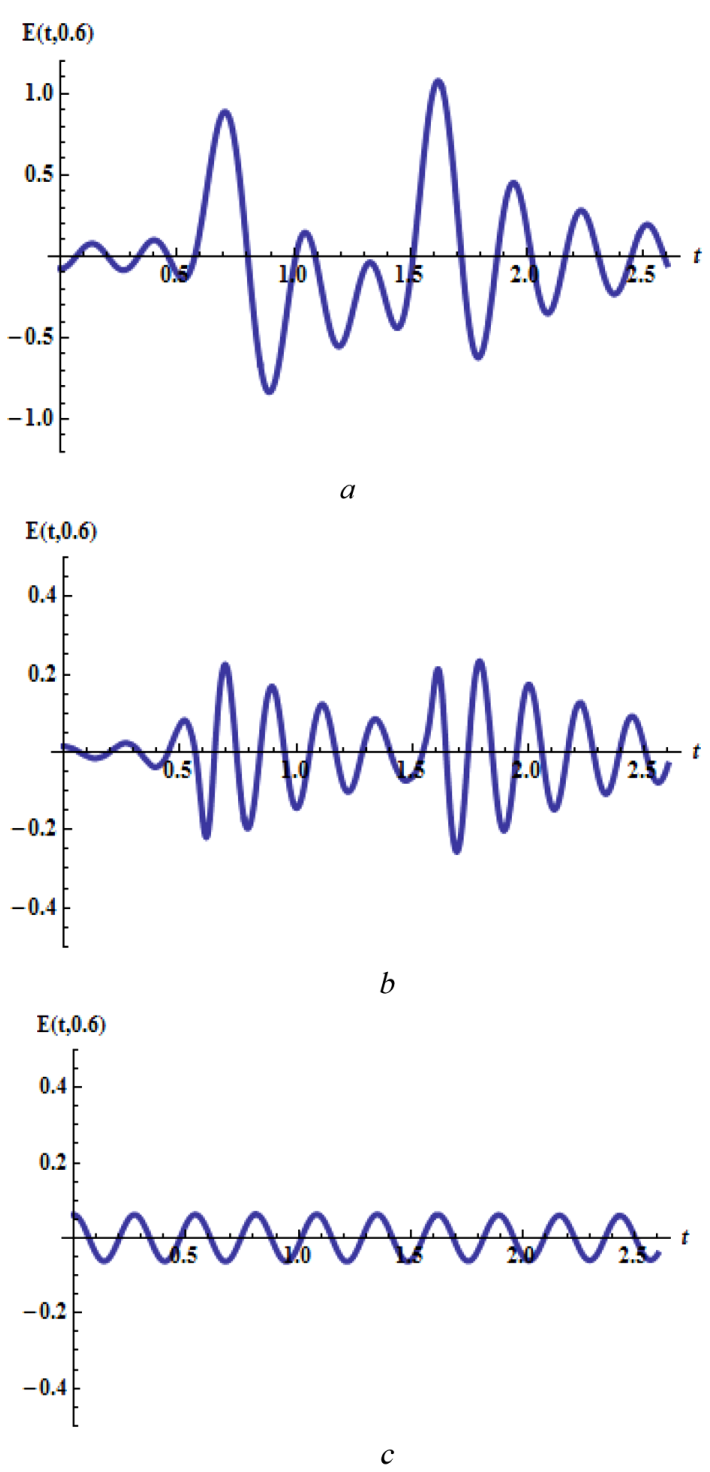

Fig. 5. Spectrum decomposition of a signal $E_{6}(t, z)$ for $z=0.6$ and the frequency band $\left\{\omega^{\min }, \omega^{\max }\right\}:\{0.23\}(a) ;\{26, \infty\}(b)$; $\{23,26\}(c): \omega_{p}=2, \omega_{0}=25, y=2$

$3 \times 10^{8} \mathrm{~m} / \mathrm{s}$ ). One can see that the peaks of a pulse have velocity that is less than $C$ for $\omega_{0}>25$ and more than $C$ for $\omega_{0}<25$.

These results correspond to the conception of group velocity $[2,3]$. Properly from the dependences $E(z, t)=C_{i}$ for $\omega_{0}=24$, the propagation velocities of peaks equal to $\simeq C$. We cannot make such conclusions for differential velocities at any point of the diagram $E(z, t) \equiv C_{i}$ (Fig. 6). 

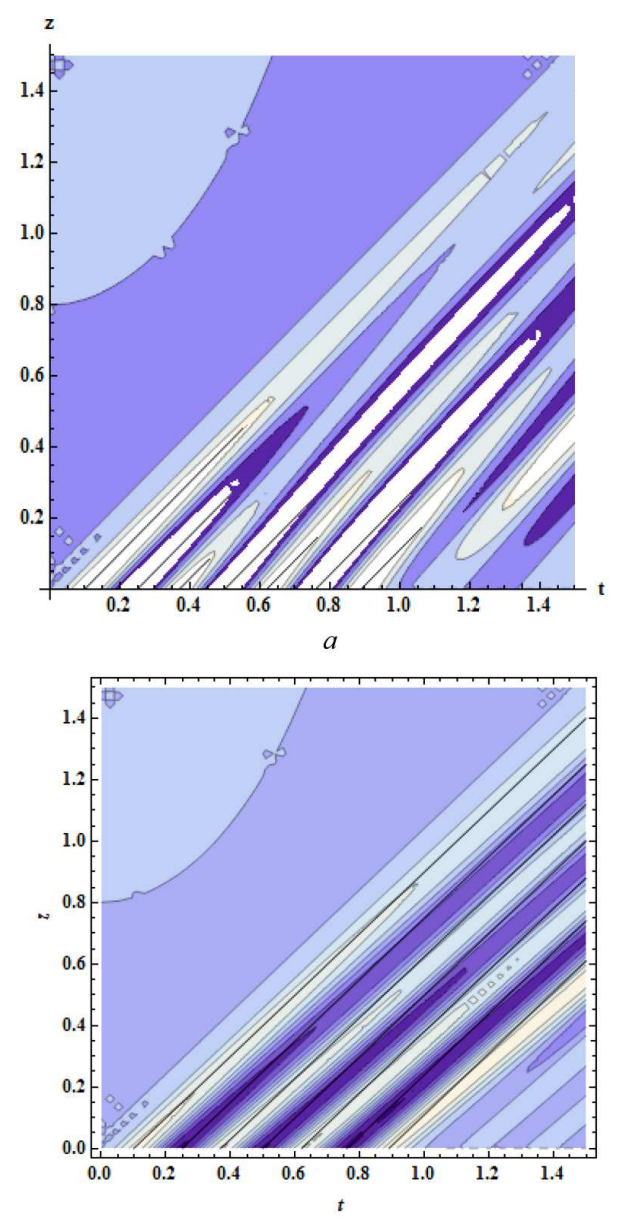

$b$

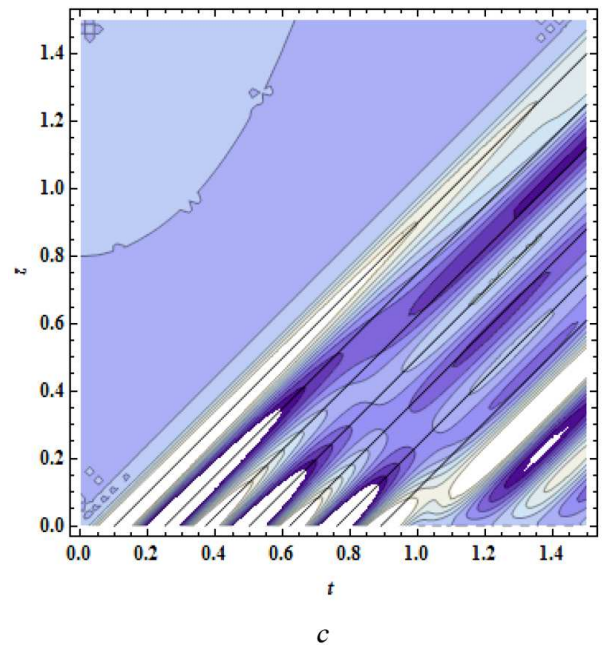

Fig. 6. Contour dependences $E_{6}(z, t)=C_{i}$ for different $\omega_{0}$ : $20(a) ; 30(b) ; 24(c): \omega_{p}=2, y=2$. Dark lines conform to the light propagation in vacuum

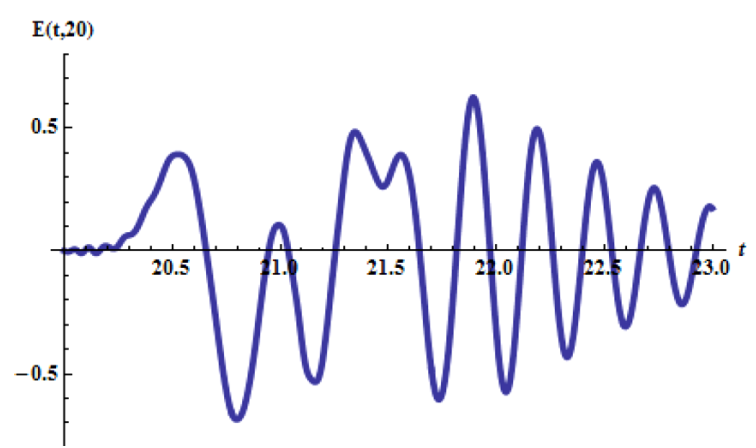

Fig. 7. EWP-pulse $E_{0}(z, t)$ for $z=20$ and a "precursor" pulse for $20<t<20.3: \omega_{p}=2, \omega_{0}=35, y=2$

As was stated by Sommerfeld and Brillouin [8, 9], the so-called "forerunner" precedes the basic pulse. In Fig. 7, one can see the example of such "forerunner".

Along with Fig. 6, we can conclude that the velocity of this forerunning pulse is equal to $C$. It is clearly that, for high frequencies, there is no absorption and dispersion. So, this small part of the input pulse propagates in a medium like in vacuum. As follows from Fig. 7, the leading edge of the pulse corresponds to $Z / C T=20$. Thus, its propagation speed is equal to the speed $C$ of light in vacuum, which characterizes it as a precursor, a forerunner pulse. The next point of this article is about the $C$-restriction on the velocity of optical pulses. The common point of view is that only the signal (or information) propagation is limited by the $C$-velocity [9-12]. We intend to demonstrate that this is true, if we take the pulse velocity and the degradation of the flow of information into account simultaneously.

\section{C-Restriction on the Data Transfer Velocity}

In the consideration of a causality, only the optical signal velocity (OSV) is restricted by the light constant $C$ in vacuum [9]. This is in agreement with the point of view of the majority of researches. As for the phase and group velocities, they may take any value. For example, there are the so-called "slow light" and "fast light" $[10,11]$. However, these notions appeared as a consequence of the slowly varying amplitude approximation (or quasimonochromatic approximation) for electromagnetic signals. Therefore, it cannot have physical significance. In this section, we propose a quantitative definition of the information spreading velocity in a dispersive medium $V_{d}$

ISSN 2071-0194. Ukr. J. Phys. 2018. Vol. 63, No. 6 
[13]. It has to take into account: (1) time delay of a signal due to the propagation; (2) duration of a signal; (3) modification of the information value; (4) time delay for a signal due to the "precursor" beforunning. The flow $J(z)$ of information on the $z$-distance is

$$
J(z)=\frac{z}{z / C+T_{p}+T} \frac{\log \left(1+\frac{S(z)}{N}\right)}{C T S},
$$

where $S(z) / N$ is the signal-to-noise ratio (SNR), $T_{p}$ is the precursor pulse duration, $T$ is the duration of a signal, and $S$ is the square of the optical beam. Flow (27) is equal to the volume concentration of the information on the entrance multiplied by OSV, which depends on the distance $V_{d}(z)$. So, we have

$V_{d}(z) \frac{\log \left(1+\frac{S_{0}}{N}\right)}{C T S}=\frac{C}{1+\frac{C}{z}\left(T_{p}+T\right)} \frac{\log \left(1+\frac{S(z)}{N}\right)}{C T S}$.

Then

$\frac{V_{d}(z)}{C}=\frac{z / C}{z / C+T_{p}+T} \frac{\log \left(1+\frac{S(z)}{N}\right)}{\log \left(1+\frac{S_{0}}{N}\right)}$.

One can see that $V_{d}(z)<C$, because both of two multipliers are less than 1 .

Now, let us calculate $T_{p}(z)$ and $S(z)$. The basic signal follows the precursor. Then the time interval $\left[T_{p}, T_{p}+T\right]$ has to fall in the time interval of the orthogonality of a signal. So,

$I\left(z, T_{p}\right)=\int_{z+T_{p}}^{z+T_{p}+1} E(z, t) d t \simeq 0$.

Equation (38) is written in normalized parameters: $z \equiv z / C T, T_{p} \equiv T_{p} / T, t \equiv t / T$. The result of a numerical solution of Eq. (38) is shown in Fig. 8. Since $T_{p} \ll T$, we can use the linear approximation $T_{p} \simeq$ $p z, p \ll 1$.

Now, we can calculate $S(z) / S_{0}$. Let us use the orthogonality for the time interval $\left[T_{p}, T_{p}+1\right]$. Then

$$
\begin{aligned}
& \alpha(z) \int_{z+T_{p}}^{z+T_{p}+1} E_{0}^{2}(t) d t=\int_{z+T_{p}}^{z+T_{p}+1} E(t, z) E_{0}(t) d t, \\
& \alpha(z)=\int_{z+T_{p}}^{z+T_{p}+1} E(t, z) E_{0}(t) d t .
\end{aligned}
$$

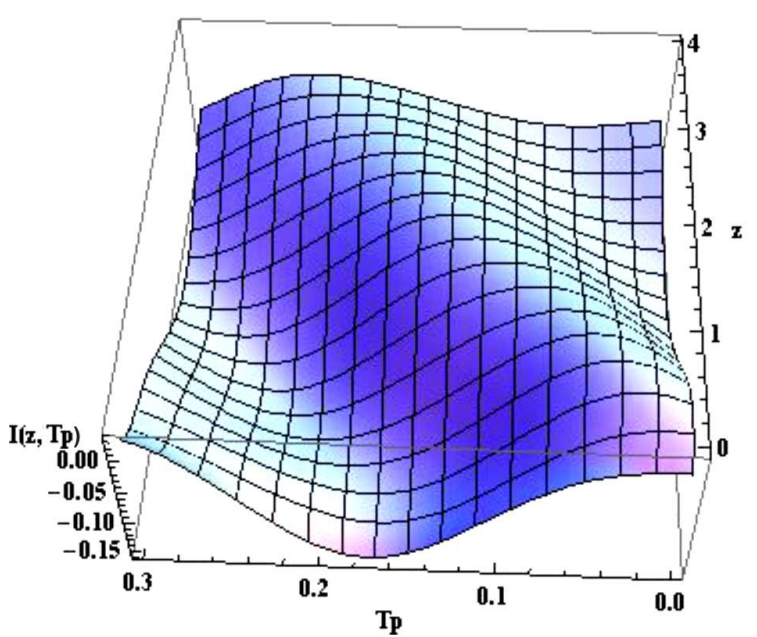

Fig. 8. Duration of the precursor pulse $T_{p}(z): \omega_{p}=2, \omega_{0}=$ $=30, y=2$. $T_{p}(z)$ is defined by the interval of orthogonality $\left[z+T_{p}, z+T_{p}+1\right]$ in $(30)$

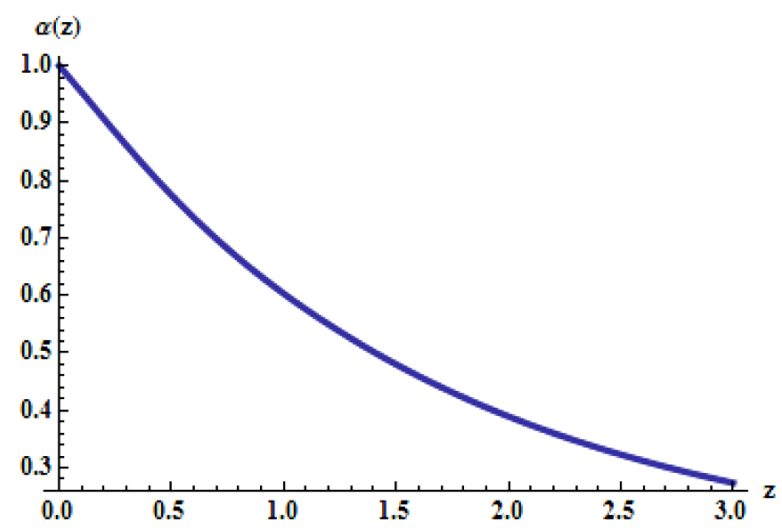

Fig. 9. Weight coefficient $\alpha(z)$ for $E_{6}(t)$ of an EWP-pulse: $\omega_{p}=2, \omega_{0}=30, y=2$

So, Eq. (29) can be written with regard for (32) (for normalized parameters) as

$\frac{V_{d}(z)}{C}=\frac{z}{z+T_{p}(z)+1} \frac{\log \left(1+\alpha(z) \frac{S_{0}}{N}\right)}{\log \left(1+\frac{S_{0}}{N}\right)}$.

The result of calculations for $\alpha(z)$ is shown in Fig. 9. A decrease of $\alpha(z)$ with growing the distance is due to the dispersion of a medium: the absorption and the signal shape degradation.

At the end of the section, let us make a remark about the causality principle. As for our case, the "causality principle" consists in reproducing the shape of a signal after it has crossed the dispersive medium. 


\section{Background for Time-Domain Spectroscopy}

Ultrawideband optical signals are well adopted for time-domain spectroscopy [14]. Let us consider the EWP optical pulse of the third order $E_{6}(t)(32)$.

The maximum amplitude of the spectral component is localed near $(\omega T)^{\max } \simeq \simeq 25$ (Fig. 6). If the duration of a signal is $T \simeq 10^{14} \mathrm{~s}$, then $\omega^{\max } \simeq$ $\simeq 2.5 \times 10^{15} \mathrm{~s}^{-1}$.
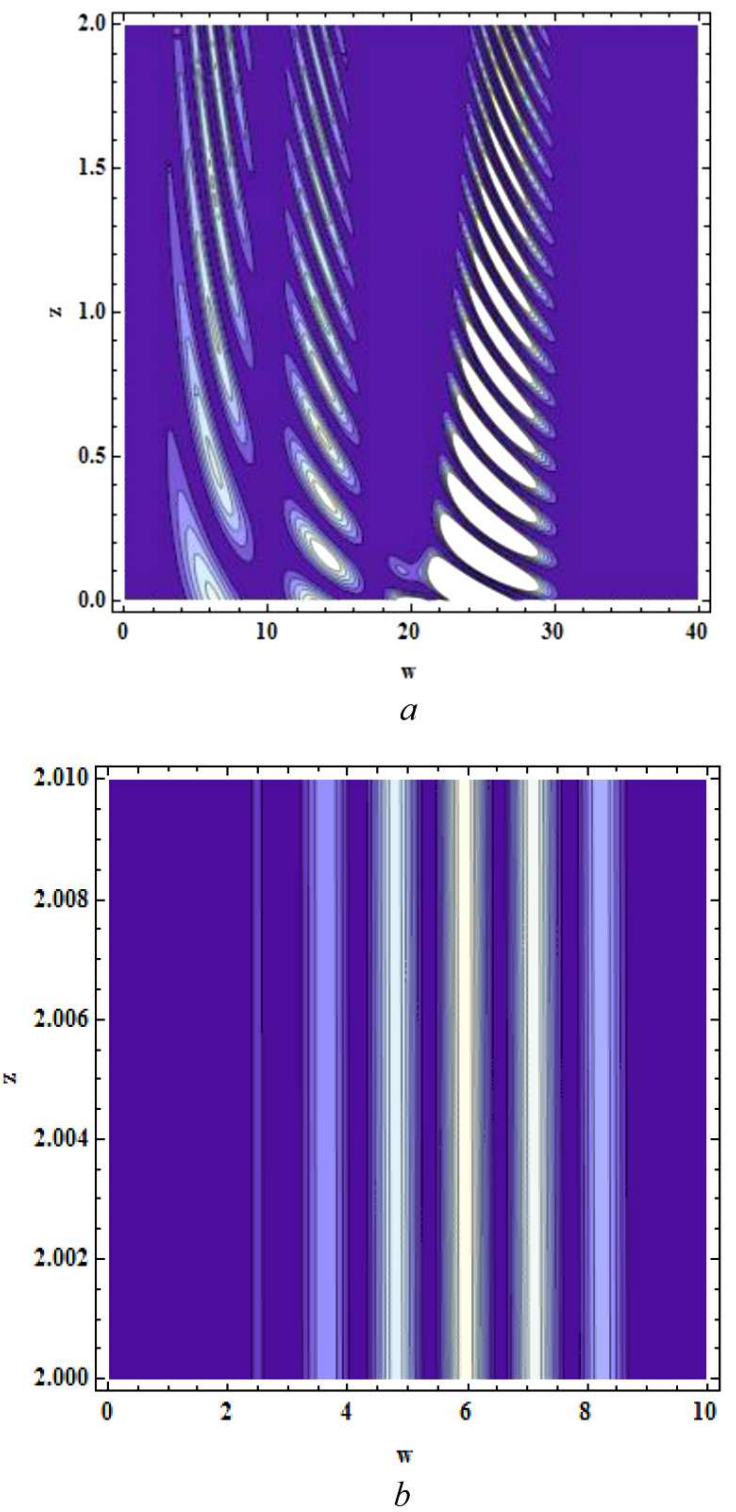

Fig. 10. Contour dependences on large $(a)$ and small $(b)$ scales $E_{6}^{2}(\omega, z)=C_{i}^{2}: \omega_{p}=2, \omega_{0}=20, y=2$
The pulse $E_{6}(t)$ propagating through a one-resonance dispersive medium has the spectrum

$E_{6}(\omega, z)=\exp [-\kappa(\omega) \omega z]\left(E_{0}(\omega) \cos [n(\omega) \omega z]-\right.$

$\left.-\frac{E_{1}(\omega)}{\omega} \sin [n(\omega) \omega z]\right)$,

where $E_{0}(\omega)$ and $E_{1}(\omega)$ correspond to formulae (33) and (34).

Let us calculate the spectrum of $E_{6}(\omega, z)$ as the contour dependence $E_{6}(\omega, z)=C_{1}$ which is a constant (Fig. 10).

So, we have to use four spectrum peaks in calculations with the simplified formula $n\left(\omega_{i}\right) \omega_{i} z=i \pi$, $i \in Z$.

We now calculate four parameters: $\omega_{p}, \omega_{0}, y$ and " $i$ " from four equations

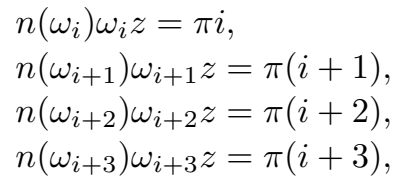

where the $n(\omega)$ dependence is shown in Eqs. (25) and (26). The exact value of $\omega_{i}$ can be measured, if we change the scale of Fig. 10, $a$ and build the dependence (Fig. 10, b)

$E_{6}^{2}(\omega, z)=C_{i}^{2}$.

One can see for $z=2: \omega_{i}=3.6, \omega_{i+1}=4.8$, $\omega_{i+2}=6, \omega_{i+3}=7.2$. So, $i=3$, and system $(43)$ reduces to three equations

$$
\begin{aligned}
& n\left(\omega_{3}\right) \omega_{3} z=3 \pi, \\
& n\left(\omega_{4}\right) \omega_{4} z=4 \pi, \\
& n\left(\omega_{5}\right) \omega_{5} z=5 \pi .
\end{aligned}
$$

To calculate $\omega_{p}, \omega_{0}$, and $y$, we have to measure frequencies with higher accuracy $\left(10^{-3}\right)$. System (45) can be solved numerically to calculate $\omega_{p}, \omega_{0}$, and $y$. To get the more precise values for the parameters $n, \omega_{p}, \omega_{0}$, and $y$, more precise data for $\omega_{i}$ and (or) optimal probe optical pulse shape are required.

\section{Conclusions}

In the investigation of optical pulse propagation, we propose a series of Elementary Waves Packets (EWP) for the representation of electromagnetic signals of any duration [6]. The EWP basic functions are superwideband signals. For the representation of a signal with a narrow spectrum, it is necessary to combine 
high-order EWP functions with certain amplitudes [15]. So, by introducing superwideband signals, we are faced with the necessity to investigate their propagation through a dispersive medium. Therefore, first of all, we solve the wave equation without any restriction on the parameters of an electromagnetic pulse and the medium. Of course, we confine ourselves by a linear nonmagnetic classical model of the medium. In addition, we do not take consider the Fresnel reflection on the both sides of the substance sample.

The characteristic property of this problem is a considerable excess of the spectral width of an optical signal above the spectral width of a medium oscillator. Therefore, we have investigated "high-frequency" and "low-frequency" oscillators. Our calculations confirm the existence of the so-called "precursor pulse" that propagates through a medium with the velocity $C$ of the light in vacuum. The duration of a precursor $T_{p}$ is equal to the delay of the orthogonal time interval $T$ for an electromagnetic pulse. The second peculiarity of a pulse is high frequencies of the pulse action on a medium like the impact to excite relaxation oscillations at the leading and trailing ends of an optical pulse. As for the propagation velocity of light, we examine the so-called optical signal velocity (OSV). As is generally assumed, only the information velocity is restricted by light velocity $C$ in vacuum. So, we have proposed a quantitative definition of OSV. It involves the time delay of the pulse propagation in a dispersive medium and information losses. It is obvious that $\mathrm{OSV}$ in vacuum is equal to $C$. The wide spectrum of an EWP-pulse is useful for its application in time-domain spectroscopy. In the last part of the present work, we will propose a method that involves the spectral peculiarities of high-order EWPpulses. It is applied to a one-resonance medium to calculate its three physical characteristics: $\omega_{p}, \omega_{0}$, and $y$ or $z$. We hope for that the results of this work will be useful in experimental studies of the propagation of short optical pulses in a dispersive medium.

The authors thank Yurii Owechko who provided helpful editing comments and suggestions.

1. M. Born, E. Wolf. Principles of Optics (Cambridge Univ. Press, 2003).

2. L.D. Landau, E.M. Lifshitz. Electromagnetics of Continuous Media (Elsevier-Butterworth-Heinemann, 2004).

3. M.B. Vinogradova, O.V. Rudenko, A.P. Sukhorukov. The Theory of Waves (Nauka, 1990) (in Russian).
4. S.A. Akhmanov, V. A. Vysloukh, A.S. Chirkin. Optics of Femtosecond Laser Pulses (American Institute of Physics, 1992).

5. Z. Chang, P.Corcum. Attosecond photon sources: The first decade and beyond. JOSA B 27, B9 (2010).

6 . V.S. Ovechko. Femtosecond optics - optics of the elementary waves packets. JOSA B 29, B799 (2012).

7. G. Arfken, H. Weber, E. Harris. Mathematical Methods for Physicists (Elsevier Academic Press, 2005).

8. L. Brillouin. Wave Propagation and Group Velocity (Academic Press, 1960).

9. P.W. Milonni. Fast Light, Slow Light and Left-Handed Light (CRC Press, 2004).

10. S.H. Chin. Governing the Speed of a Light Signal in Optical Fibers: Brillouin Slow and Fast Light. Thesis. No. 4459, EPFL (2009).

11. M.D. Stenner, D.I. Gauthier, M.A. Neifeld. The speed of information in a "fast light" optical medium. Nature $\mathbf{4 2 5}$ (6959), 695 (2003).

12. N. Brunner, V. Scarani, M. Wegmuller, M. Legre, N. Gisin. Direct measurement of superluminal group velocity and signal velocity in an optical fiber. Phys. Rev. Lett. 93 (20), (2004).

13. V. Ovechko. Femtosecond optical pulse propagation through the single resonance Lorentz model dielectric. Int. J. ARPS 4 (1), 28 (2017).

14. V.S. Ovechko, V.P. Myhashko, S.O. Motovilovetz. Elementary wave packets decomposition - new spectroscopy technique, in Book of Abstracts of the XXII Galina Puchkovska International School Seminar on Spectroscopy of Molecules and Crystals, Chynandiyovo, Ukraine, September 27 - October 4, 2015, p. 280.

15. V.S. Ovechko, V.P. Myhashko, A.A. Kornienko. Slowly varying amplitude approximation in optics, in Proceeding of the XI International Conference "Electronics and Applied Physics", Taras Shevchenko National University of Kyiv, Ukraine, October 21-24, 2015, p. 20.

Received 10.01.18

В.С. Овечко, В.П. Мигашко

СПЕКТРАЛЬНІ ОСОБЛИВОСТІ

ПОШИРЕННЯ ФЕМТОСЕКУНДНИХ ОПТИЧНИХ ІМПУЛЬСІВ У ДИСПЕРСІЙНОМУ СЕРЕДОВИЩІ

Р е $з$ ю м е

Запропоновано уточнений розв'язок хвильового рівняння для диспергуючого середовища без обмежень на тривалість оптичного імпульсу. Застосовано розклад в ряд елементарних хвильових пакетів (ЕХП) для опису надширокосмугових фемтосекундних (фс) оптичних імпульсів Досліджено особливості поширення низьких та високих частот в однорезонансному середовищі. Показано існування "прекурсора" для фс оптичних імпульсів. Запропоновано формулу для швидкості поширення оптичного сигналу. Вона не перевищує швидкості світла у вакуумі. Запропоновано методику використання ЕХП - імпульсів в "time-domain" спектроскопії. 